\title{
Hard X-ray emitting energetic electrons and photospheric electric currents
}

\author{
S. Musset, N. Vilmer, and V. Bommier \\ LESIA, Observatoire de Paris, CNRS, UPMC, Université Paris-Diderot, 5 place Jules Janssen, 92195 Meudon, France \\ e-mail: sophie.musset@obspm.fr
}

Received 11 June 2014 / Accepted 19 June 2015

\begin{abstract}
Context. The energy released during solar flares is believed to be stored in non-potential magnetic fields associated with electric currents flowing in the corona. While no measurements of coronal electric currents are presently available, maps of photospheric electric currents can now be derived from SDO/HMI observations. Photospheric electric currents have been shown to be the tracers of the coronal electric currents. Particle acceleration can result from electric fields associated with coronal electric currents. We revisit here some aspects of the relationship between particle acceleration in solar flares and electric currents in the active region.

Aims. We study the relation between the energetic electron interaction sites in the solar atmosphere, and the magnitudes and changes of vertical electric current densities measured at the photospheric level, during the X2.2 flare on February 15, 2011, in AR NOAA 11158. Methods. X-ray images from the Reuven Ramaty High Energy Solar Spectroscopic Imager (RHESSI) are overlaid on magnetic field and electric current density maps calculated from the spectropolarimetric measurements of the Helioseismic and Magnetic Imager (HMI) on the Solar Dynamics Observatory (SDO) using the UNNOFIT inversion and Metcalf disambiguation codes. X-ray images are also compared with extreme ultraviolet (EUV) images from the SDO Atmospheric Imaging Assembly (AIA) to complement the flare analysis.

Results. Part of the elongated X-ray emissions from both thermal and non-thermal electrons overlay the elongated narrow current ribbons observed at the photospheric level. A new X-ray source at $50-100 \mathrm{keV}$ (produced by non-thermal electrons) is observed in the course of the flare and is cospatial with a region in which new vertical photospheric currents appeared during the same period (an increase of 15\%). These observational results are discussed in the context of the scenarios in which magnetic reconnection (and subsequent plasma heating and particle acceleration) occurs at current-carrying layers in the corona.
\end{abstract}

Key words. Sun: flares - Sun: particle emission - Sun: X-rays, gamma rays - Sun: magnetic fields - acceleration of particles

\section{Introduction}

It is now commonly admitted that solar flares are the result of the sudden release of magnetic free energy in the corona, this free energy being stored in non-potential magnetic fields associated with electric currents flowing in the corona. As there are currently no measurements of the vector magnetic fields in the corona, there are also no measurements of coronal electric currents. Electric currents have only been determined in a few cases at the photospheric level using vector magnetic field measurements achieved in photospheric lines. While some pioneering work was done in the 1970 s by e.g. Moreton \& Severny (1968) at the Crimean Astrophysical Observatory, many observations of vector magnetic fields and related electric currents were obtained later in the 1980s and 1990s with ground-based vector magnetographs (see e.g. Hagyard et al. 1984; Canfield et al. 1993). Nowadays, the polarimetric measurements obtained continuously and with an unprecedented cadence and spatial resolution with the Helioseismic and Magnetic Imager (HMI) on Solar Dynamics Observatory (SDO) provides maps of photospheric vertical electric currents for flaring active regions and also allows the study of the evolution of these currents on a time resolution of $12 \mathrm{~min}$ (see e.g. Petrie 2012, 2013; Janvier et al. 2014).

The relation between the energetic phenomena (plasma heating and particle acceleration) occurring in solar flares and the electric current systems in active regions has been the subject of many studies for several decades. In particular, the link between the photospheric electric currents and the energetic electron precipitation sites has been investigated in several events, originally to discuss the relevance of the different flare models presented in the literature. The first studies used $\mathrm{H} \alpha$ observations to characterize electron precipitation sites since no hard X-ray (HXR) imaging observations were available. Using data from the Crimean Astrophysical Observatory, Moreton \& Severny (1968) examined the spatial relationship between $\mathrm{H} \alpha$ flare kernels and maxima of vertical electric current densities. For $80 \%$ of the 25 events studied, a spatial coincidence $\left(<6^{\prime \prime}\right)$ was found between the location of the centre of the bright $\mathrm{H} \alpha$ kernel and the location of the strongest electric current densities $\left(>8 \mathrm{~mA} / \mathrm{m}^{2}\right)$. Such a spatial coincidence between $\mathrm{H} \alpha$ flare kernels and strong electric current densities was later confirmed by other studies. Lin \& Gaizauskas (1987) found in particular that the sites of strongest $\mathrm{H} \alpha$ emissions were cospatial with the vertical current systems at the photospheric level to within $2^{\prime \prime}$. However, it was also found that even if some of the flare kernels were close to the electric currents, many of them appeared near the edges of currents (Romanov \& Tsap 1990). In a series of papers, Canfield et al. (1993), Leka et al. (1993), de La Beaujardiere et al. (1993) revisited the question of the relation between electric current systems and electron precipitation sites using vector magnetograph data from the Mees Solar Observatory and information from $\mathrm{H} \alpha$ line profiles that allow $\mathrm{H} \alpha$ signals to be disentangled 
from electron precipitation and from high pressure. Studies of several flares (Leka et al. 1993; de La Beaujardiere et al. 1993) confirmed that sites of intense non-thermal electron precipitation do not coincide with the regions of strongest vertical currents at the photosphere, but tend to occur near channels of high vertical current density rather than at the vertical current density maxima. In a further study, Demoulin et al. (1997) found for several events that flare ribbons were located in the vicinity of strong electric currents in the photosphere. They furthermore derived the locations of the photospheric footprints of regions of rapid change in magnetic line connectivity called quasi-separatrix layers (QSLs). They found, as in other observational studies (see e.g. Machado et al. 1983; Mandrini et al. 1995; Bagalá et al. 1995), that flares tend to occur near the locations of these QSLs. These QSLs are now known to be regions where strong current densities can develop and where reconnection can occur (see e.g. Demoulin et al. 1996; Aulanier et al. 2005), thus establishing a clear link between flare energy release, electric currents and magnetic reconnection.

With the arrival of HXR imagers, the link between vertical electric currents at the photosphere and electron precipitation sites has been revisited using in particular YOHKOH/HXT observations (see Canfield et al. 1992; Li et al. 1997). The latter study based on six events confirms that HXR emission and thus electron precipitation sites are not exactly cospatial with regions of highest vertical current densities at photospheric levels, but instead are adjacent to the current channels, therefore confirming the results obtained by de La Beaujardiere et al. (1993) with $\mathrm{H} \alpha$ observations. In a later paper, Aschwanden et al. (1999) showed how this offset between the maximum of vertical currents and the HXR loop footpoints could be explained in the context of the 3D geometry of quadrupolar reconnection. It must finally be noted that in the earlier studies, vector magnetograms (and thus electric current maps) were sparsely derived and that the integration time to derive such information could be more than one hour. Several hours may also separate the time of the flare and the time of the magnetic field measurements. Nowadays, the combination of polarimetric measurements continuously obtained with the HMI on SDO and of HXR observations of solar flares obtained with the Reuven Ramaty High Energy Solar Spectroscopic Imager (RHESSI) allows the comparison of high quality vector magnetic field (and electric current) maps at a cadence of 12 min and HXR observations of solar flares at exactly the same time. This provides new possibilities to study more systematically and in greater detail the relation between the location of HXR sources (and electron precipitation sites) and the vertical electric current densities.

This paper presents the first results of such a study for the flare of February 15, 2011. The simultaneous evolutions of electric currents and of HXR emission sources during the flare are examined for the first time. The February 15, 2011, event is quite appropriate for such a first study. Indeed, this major flare (GOES class X2.2) occurred in the active region AR11158 when the active region was near disk-centre. The derivation of vector magnetic field maps and of vertical electric current densities requires observations of an active region located near the centre of the solar disk to have good measurements of the vector magnetic field at the photospheric level and to avoid projection effects. The February 15, 2011, flare which is the first X-class flare of cycle 24 has been the subject of numerous studies since the original analysis by Schrijver et al. (2011; see e.g. for some of the most recent papers Inoue et al. 2014, for MHD simulations of the flare). In close relationship with the present paper, Janvier et al. (2014) quantified the vertical currents at the photospheric level and studied their temporal evolution during the flare. They concluded in particular that the evolutions of current and flare ribbons are related to the evolution of the magnetic field during the flare in the context of a 3D standard flare model. The active region itself and its activity has also been extensively studied. In particular, Sun et al. (2012), Vemareddy et al. (2012), Aschwanden et al. (2014), Zhao et al. (2014) realized different estimations, for example of the free magnetic energy and dissipated energy for several events occurring in this active region.

In Sect. 2 of this paper, we present briefly the different instruments used in this study as well as the techniques necessary to obtain maps of vector magnetic fields and electric currents from the measurements. In Sect. 3, X-ray and EUV observations of the flare are compared with the vertical electric current densities. Section 4 discusses the observational results and presents our interpretation. A summary of the paper and of the conclusions is presented in Sect. 5.

\section{Magnetic field measurements and flare observations}

\subsection{Magnetic field measurements and electric current calculations}

The magnetic field and electric current density maps are derived from the data of the HMI on SDO (Scherrer et al. 2012; Schou et al. 2012). The HMI instrument provides images of the entire Sun in six narrow spectral bands in a single iron line (FeI $617.33 \mathrm{~nm}$ ) and in four different states of polarization. This set of 24 images provides spectropolarimetric data to enable the calculation of the full vector magnetic field (inverse problem), at the altitude of formation of the spectral line, which is at the photospheric level in this case. The three components of the photospheric vector magnetic field can be calculated together with the vertical component of the electric current density (see e.g. Fig. 1 bottom and Janvier et al. 2014, for further details). However, because the spectropolarimetric measurements are performed in only one single line (i.e. at one altitude), it is not possible to derive all the components of the electric current density but only the vertical one. The spatial resolution is 0.91 arcsec and one map of the vector magnetic field and of the vertical electric current can be calculated at a 12 min time cadence.

The details of the calculation of magnetic field and vertical density for this set of data is described in Janvier et al. (2014). Level-1b IQUV data were used for inversion with the MilneEddington inversion code UNNOFIT presented in Bommier et al. (2007). The specificity of UNNOFIT is that, in order to take into account the unresolved magnetic structures, a magnetic filling factor is introduced as a free parameter of the LevenbergMarquardt algorithm that fits the observed set of profiles with a theoretical one. However, for further application we use only the averaged field, i.e. the product of the field by the magnetic filling factor, as recommended by Bommier et al. (2007). The interest of the method lies in a better determination of the field inclination. This is of major importance for our study since the currents are determined from the horizontal components of the magnetic field.

After the inversion, the $180^{\circ}$ remaining azimuth ambiguity was resolved by applying the ME0 code developed by Metcalf, Leka, Barnes, and Crouch (Leka et al. 2009) ${ }^{1}$. After resolving the ambiguity, the magnetic field vectors were rotated into the local reference frame (i.e. in the heliographic reference frame,

Available at http://www. cora.nwra.com/AMBIG/ 
see Fig. A.1), where the local vertical axis is the $O z$ axis (perpendicular to the solar surface at the centre of the frame). The vertical component of the electric current density (perpendicular to the plane of photosphere, i.e. in the heliographic coordinates) was then calculated via the curl of the magnetic field.

\subsection{Flare observations}

The RHESSI measures HXR and gamma-ray emissions from the Sun in the $3 \mathrm{keV}-10 \mathrm{MeV}$ range (Lin et al. 2002). Equipped with nine rotating collimators (pairs of identical grids in front of each detector), it provides images (Hurford et al. 2002) in addition to spectra (Smith et al. 2002). The spatial resolution is determined by the pitch of the grids of the collimators used to reconstruct the image. In this paper images were obtained using the CLEAN and Visibility Forward Fit algorithms using all the collimators except the first one (smallest pitch): the spatial resolution is then of 3.9 arcsec.

The Atmospheric Imaging Assembly (AIA) instrument (Lemen et al. 2012) aboard the SDO provides images of the solar chromosphere and corona in EUV in six different wavelengths. The spatial resolution is 1.5 arcsec and for each wavelength, the time cadence is of $12 \mathrm{~s}$, which enables the detailed study of the evolution in the flare of magnetic structures and flaring plasma. As the X2.2 flare on February 15, 2011, was very intense, most of the AIA channels are saturated around the time of the peak of the flare, even with the minimal exposure time. Only the channels at $94 \AA$ and $335 \AA$ are not saturated at the time of the flare and are therefore used for comparison with X-ray and magnetic observations.

While magnetic fields and current densities are calculated in the heliographic coordinates, RHESSI and AIA images are obtained in the plane-of-sky. Therefore, to combine magnetic field maps with RHESSI images, for example, it is necessary to represent the magnetic fields and current densities in the plane-of-sky coordinates. Figure A.1 shows the geometry associated with the different measurements, and the change of coordinates to compare the different observations is described in Appendix A. One map of the full vector magnetic field and of the vertical currents represented in the plane-of-sky is shown in Fig. 1; we note that the components of the field and vertical current density are not changed in this process.

\section{Observational results}

\subsection{Temporal and spatial evolution of the $X$-ray emissions}

The X2.2 flare on Feburary 15, 2011, was detected by RHESSI in the 3-100 keV energy range. The RHESSI corrected count rates are presented in Fig. 2, together with the X-ray flux from GOES. In this figure the count rates are corrected from the changes of attenuator state during the flare; indeed, there was no attenuator (attenuator state A0) until around 01:47:10 UT, then one attenuator (A1) was in front of the detectors, and finally at 01:49:40 UT two attenuators (A3) were in place. At 02:08:50 UT, only the first attenuator was left in place. The peak of the X-ray emission in the $12-25 \mathrm{keV}$ range is around 01:54 UT.

While the temporal evolution of the count rate between 12 and $50 \mathrm{keV}$ is relatively smooth, the emission between 50 and $100 \mathrm{keV}$ is more structured. Nevertheless, the different peaks of emission in the 50-100 keV band have counterparts visible in the $25-50 \mathrm{keV}$ range. Images created in the $12-25 \mathrm{keV}$, 25-50 keV, and 50-100 keV energy bands, for different peaks
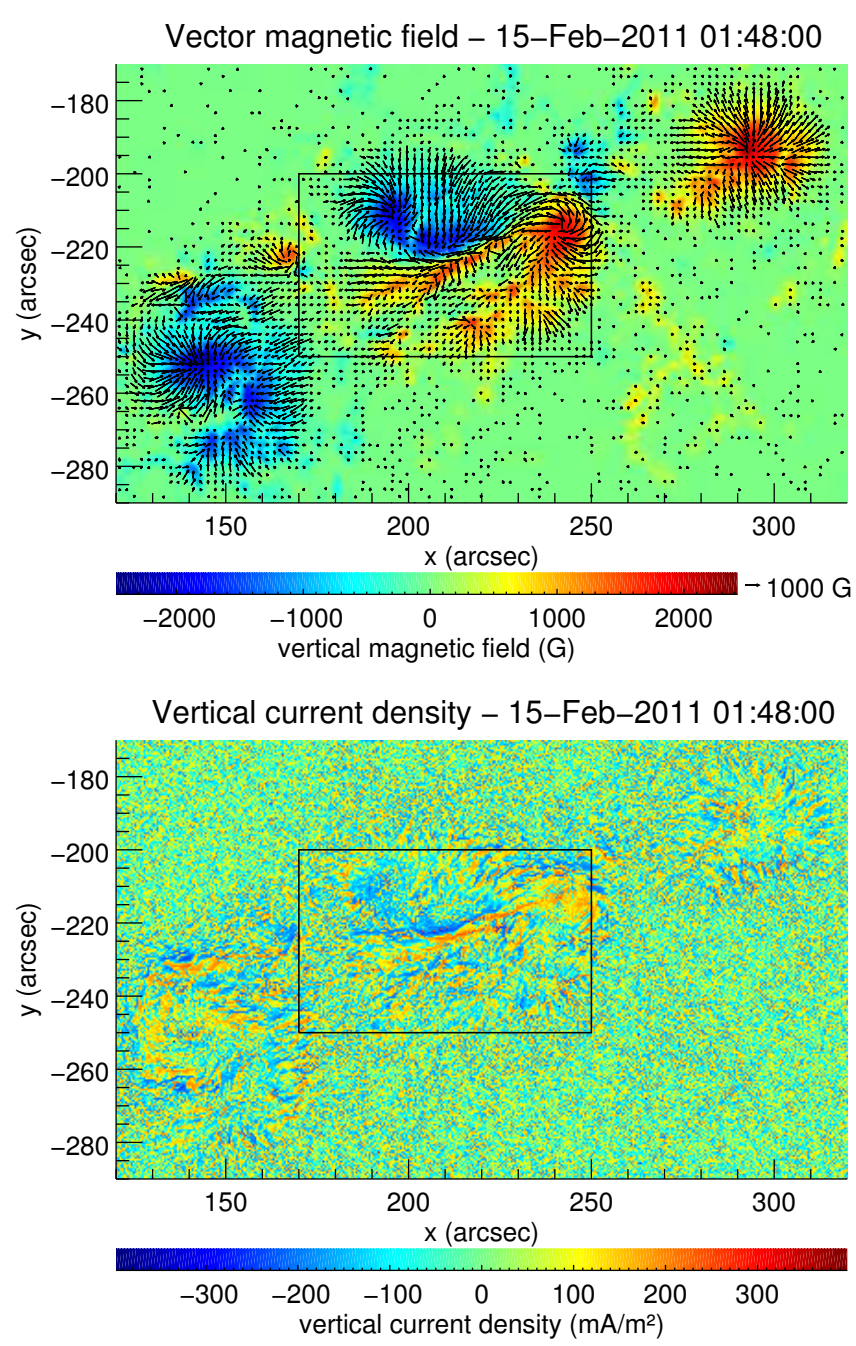

Fig. 1. Top: vector magnetic field map, bottom: vertical electric current density map of the active region NOAA AR 11158; produced from HMI data, on February 15, 2011, at 01:48:00 UT, and represented in the plane-of-sky (see text and Appendix A for more details). The rectangular box represents the field of view used in the present study. Top: the arrows represent the horizontal component of the field (for horizontal component greater than $100 \mathrm{G}$ ), and the colours represent the intensity of the vertical magnetic field (see scale). Bottom: vertical component of the electric current density (see colour scale).

of the 50-100 keV count rate, are shown in Fig. 3. We used an integration time of $16 \mathrm{~s}$, and detectors $2 \mathrm{~F}$ to $9 \mathrm{~F}$. The first set of images (first time interval) is in the attenuator state A1, whereas the other images are in the attenuator state A3.

Figure 3 shows that the X-ray emission sources both in the $12-25 \mathrm{keV}$ and $25-50 \mathrm{keV}$ energy ranges (in green and blue) have an elongated shape (more than 30 arcsec long) with several local maxima in space; the two main maxima are clearly visible in the images at $25-50 \mathrm{keV}$ for the last four time intervals in Fig. 3. The $12-25$ and $25-50 \mathrm{keV}$ emissions keep this elongated form during most of the flare. Moreover, the $25-50 \mathrm{keV}$ emission is more extended in size than that at $12-25 \mathrm{keV}$, in each time interval. This has been verified first by measuring the length of the X-ray sources using the $50 \%$ contours in the CLEAN images, as illustrated in Fig. 4 (left). To better quantify the size of the X-ray source, the visibility forward fitting technique was also used (see Schmahl et al. 2007 for the definition of visibilities; and $\mathrm{Xu}$ et al. 2008 for examples of application). This algorithm 


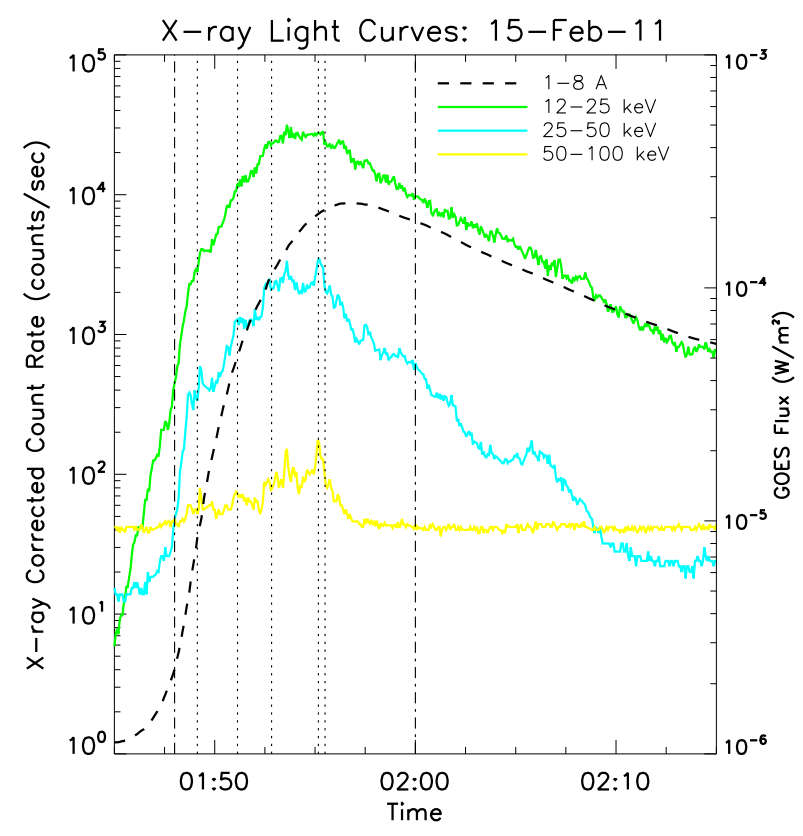

Fig. 2. RHESSI corrected count rates between 01:45 and 02:15 UT, for different energy ranges (green: $12-25 \mathrm{keV}$; cyan: $25-50 \mathrm{keV}$; yellow: $50-100 \mathrm{keV}$ ) and GOES flux between 1.0 and $8.0 \AA$ (dashed line). The vertical dash-dotted lines at 01:48 and 02:00 UT represent the time of the two magnetic maps used in the study, and the five vertical dotted lines represent the mean times when X-ray images are produced.

Table 1. Loop lengths (in arcseconds) inferred from the visibility forward fit algorithm for $40 \mathrm{~s}$ time intervals centred around (b) and (c).

\begin{tabular}{lcccc}
\hline \hline & $12-18 \mathrm{keV}$ & $18-26 \mathrm{keV}$ & $26-40 \mathrm{keV}$ & $40-60 \mathrm{keV}$ \\
\hline (b) $28.8 \pm 1.4$ & $32.6 \pm 1.0$ & $40.4 \pm 1.8$ & $44.5 \pm 4.3$ \\
(c) $28.8 \pm 0.85$ & $29.4 \pm 1.3$ & $46.3 \pm 1.9$ & $48.5 \pm 3.4$ \\
\hline
\end{tabular}

allows the size of the source to be computed and also the error on the size once a model has been chosen. In order to get sufficient statistics, images were created with a $40 \mathrm{~s}$ integration time (instead of the $16 \mathrm{~s}$ time in Fig. 3). The result of the visibility forward fit reconstruction using a curved elliptical Gaussian loop (Xu et al. 2008) as a model is shown in Fig. 4 (right column) for the interval 01:50:48-01:51:28 UT centred around interval (b).

To better determine the evolution of the length of the X-ray source with energy, images were built in narrower energy bins. It is finally found that for the intervals centred around (b) and (c), the length of the source increases logarithmically with energy with a slope of $0.23 \pm 0.03$ and $0.60 \pm 0.20$ (see Table 1), as already observed in some flares (Xu et al. 2008).

At higher energies (50-100 keV) the X-ray emission comes from compact sources: A and B visible in intervals (a), (b), and (c) and sources D and E in intervals (d) and (e). In intervals (a) to (c), sources A, B, and $\mathrm{B}^{\prime}$ are localized along the elongated structure seen at lower energies. While sources B and $\mathrm{B}^{\prime}$ seem to be located at a footpoint of the elongated sources observed at $12-50 \mathrm{keV}$, source A is located close to the middle of this structure. Between intervals (a) and (c), it can be seen that source B has moved about 9 arcsec to the north-west (source $\mathrm{B}^{\prime}$ ), while source A has moved around 3 arcsec towards the west.

A more noticeable change of configuration of the 50-100 keV sources occurs between intervals (c) and (d) when sources $\mathrm{A}$ and $\mathrm{B} / \mathrm{B}^{\prime}$ disappear and two new sources, $\mathrm{E}$ and $\mathrm{D}^{\prime}$
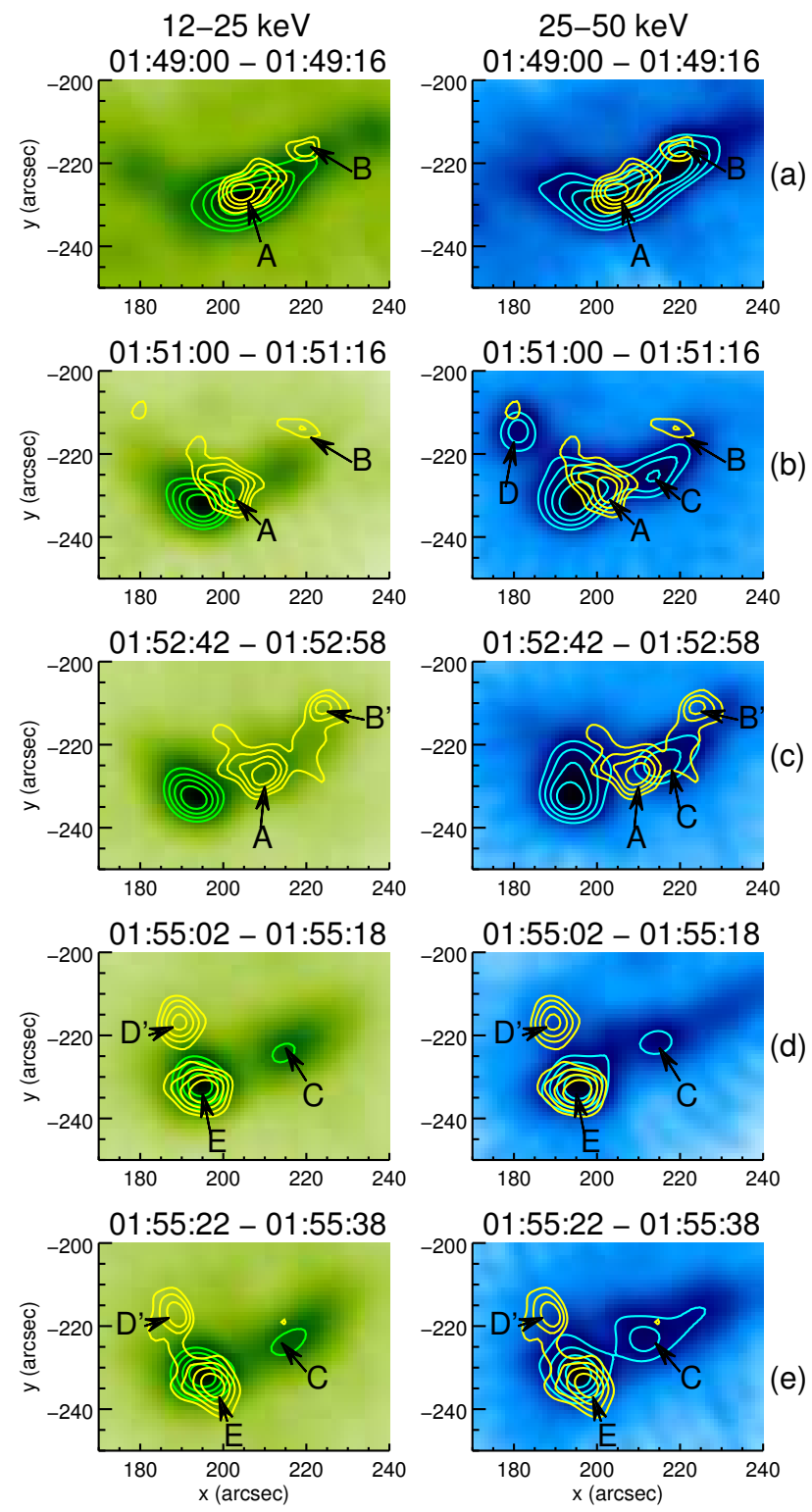

Fig. 3. Left: RHESSI images at $12-25 \mathrm{keV}$ (green), with contours at 12-25 keV (green) and 50-100 keV (yellow) overlaid on the images. Right: RHESSI images at $25-50 \mathrm{keV}$ (blue), with contours at 25-50 keV (blue) and 50-100 keV (yellow) overlaid on the images. Images and contours are shown for five time intervals integrated over $16 \mathrm{~s}$ (from top to bottom: 01:49:00-01:49:16, 01:51:00-01:51:16, 01:52:42-01:52:58, 01:55:02-01:55:18, and 01:55:22-01:55:38 UT) corresponding to peaks in the $50-100 \mathrm{keV}$ range. Images are obtained using detectors $2 \mathrm{~F}, 3 \mathrm{~F}, 4 \mathrm{~F}, 5 \mathrm{~F}, 6 \mathrm{~F}, 7 \mathrm{~F}, 8 \mathrm{~F}$, and $9 \mathrm{~F}$ and the CLEAN algorithm. The green, blue, and yellow contours are $60 \%, 70 \%, 80 \%$, and $90 \%$ of the image maximum value.

appear. Furthermore, the two sources seem to be footpoints of a loop perpendicular to the elongated structure seen at $12-50 \mathrm{keV}$. It can be seen that source $\mathrm{E}$ at $50-100 \mathrm{keV}$ is cospatial with some local maximum emission at $12-25$ and $25-50 \mathrm{keV}$ and that at the same position of source $\mathrm{D}^{\prime}$, a source D has also appeared in interval (b), but in the $25-50 \mathrm{keV}$ energy range.

The spectral analysis (performed using the two functions vth and thick 2 in the RHESSI spectral analysis software) shows that the emission is a combination of a thermal component with a 
S. Musset et al.: Hard X-ray emitting energetic electrons and photospheric electric currents
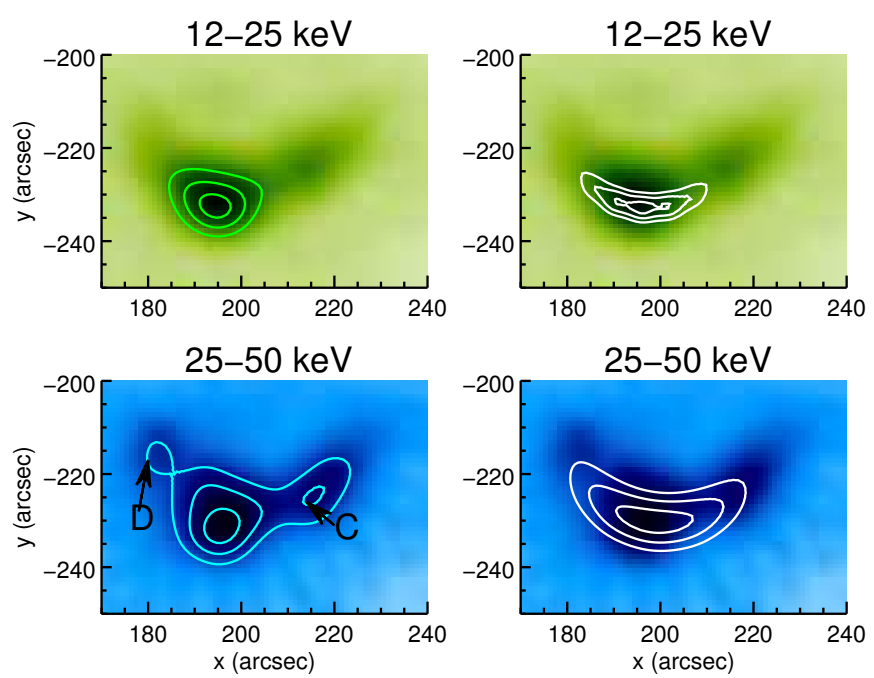

Fig. 4. RHESSI images and contours at $12-25 \mathrm{keV}$ (green) and 25-50 keV (cyan) obtained between 01:50:48 and 01:51:28 UT using two different methods. Left: CLEAN images and contours at 50\%, 70\%, and $90 \%$ of the maximum. Right: CLEAN images and contours reconstructed with the visibility forward fit technique $(50 \%, 70 \%$, and $90 \%$ of the maximum).

temperature in the $25-35 \times 10^{6} \mathrm{~K}$ range and of a non-thermal component produced by energetic electrons. There is a hardening of the non-thermal electron spectrum starting from interval (d), as the electron spectrum spectral index $\delta$ evolves from $[-7$, $-6.5]$ in intervals (a), (b), and (c) to $[-5,-4.6]$ in intervals (d) and (e). For the time intervals of the images shown in Fig. 3, the spectral analysis shows that while most of the emission in the $12-25 \mathrm{keV}$ range is of thermal origin, the emission in the 25-50 keV range is mostly produced by non-thermal electrons (the proportion of non-thermal emission in this energy range being greater than $75 \%$ in all images).

\subsection{Comparison with the EUV data from SDO/AIA}

Emissions in both $94 \AA$ and $335 \AA$ channels at around 01:51 UT are shown in Fig. 5 (middle and top panels, respectively). While the $94 \AA$ emission shows an elongated structure together with more compact sources at the edge, at $335 \AA$ most of the emission arises from the compact sources. Figure 5 (middle) also shows that some structures are emitting in both $94 \AA$ and $335 \AA$ channels (yellow contours), whereas other are emitting only at $94 \AA$ (red contours). This can be interpreted considering the different channel response to source temperature. Table 1 from Lemen et al. (2012) gives the characteristic emission temperatures of the plasma for the $94 \AA$ and $335 \AA$ channels: $6.3 \times 10^{6} \mathrm{~K}$ and $2.5 \times 10^{6} \mathrm{~K}$, respectively. However, as shown in Fig. 13 of Lemen et al. (2012), the different channels are sensitive to a wide distribution of temperatures. From this figure it is deduced that if a structure is bright at $94 \AA$ and $335 \AA$, the plasma temperature is most probably around $1 \times 10^{6} \mathrm{~K}$ (yellow contours), while if it is bright only in the $94 \AA$ channel, it would be much higher (more than $6 \times 10^{6} \mathrm{~K}$, red contours). The superposition of the elongated part of the X-ray sources below $50 \mathrm{keV}$ with the elongated structure seen at $94 \AA$ in Fig. 5 (bottom) strongly supports this interpretation since the spectral X-ray analysis shows that the X-ray emission below $50 \mathrm{keV}$ is partly emitted by a hot plasma with a
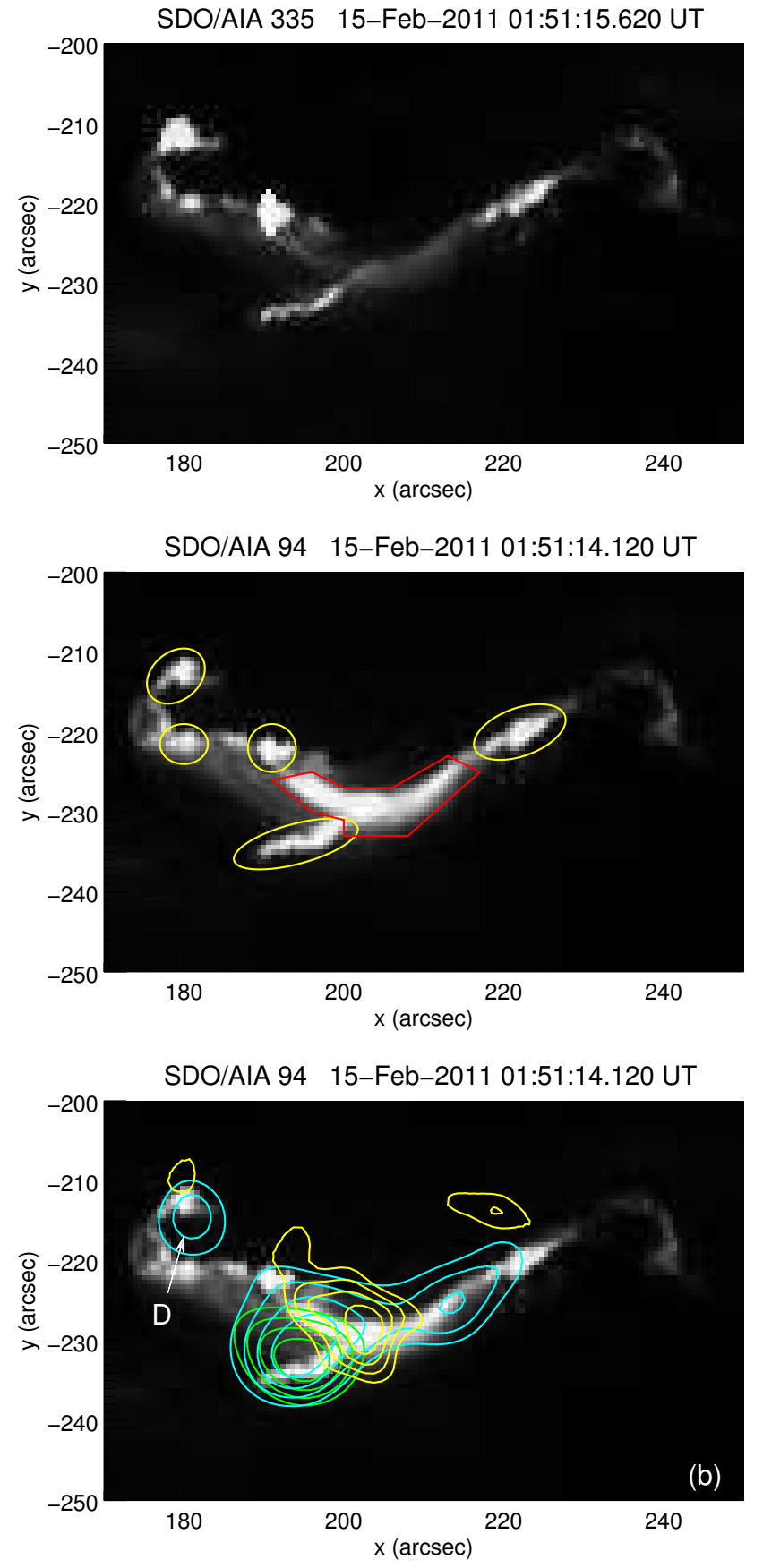

Fig. 5. SDO/AIA image at $335 \AA$ (top) and $94 \AA$ (middle and bottom), on February 15, 2011, around 01:51:15 UT. In the middle image, the region bordered in red is not clearly detected at $335 \AA$ (most probably the plasma temperature is higher than $6 \times 10^{6} \mathrm{~K}$; see text); regions bordered in yellow are seen in both channels (probably the plasma temperature is close to $1 \times 10^{6} \mathrm{~K}$ ). Bottom: image at $94 \AA$ on which RHESSI contours are overlaid. The green, blue, and yellow contours are the RHESSI emissions at $12-25 \mathrm{keV}, 25-50 \mathrm{keV}$, and $50-100 \mathrm{keV}$, respectively, integrated between 01:51:00 and 01:51:16 UT. The contour levels are similar to the ones in Fig. 3.

temperature in the range $25-35 \times 10^{6} \mathrm{~K}$. Figure 5 (bottom) also shows that source D in image (b) in Fig. 3 is roughly cospatial with a EUV source detected both at $335 \AA$ and $94 \AA$. This source is most probably the footpoint of coronal loops in which energetic electrons are injected. Figure 6 shows the evolution with 

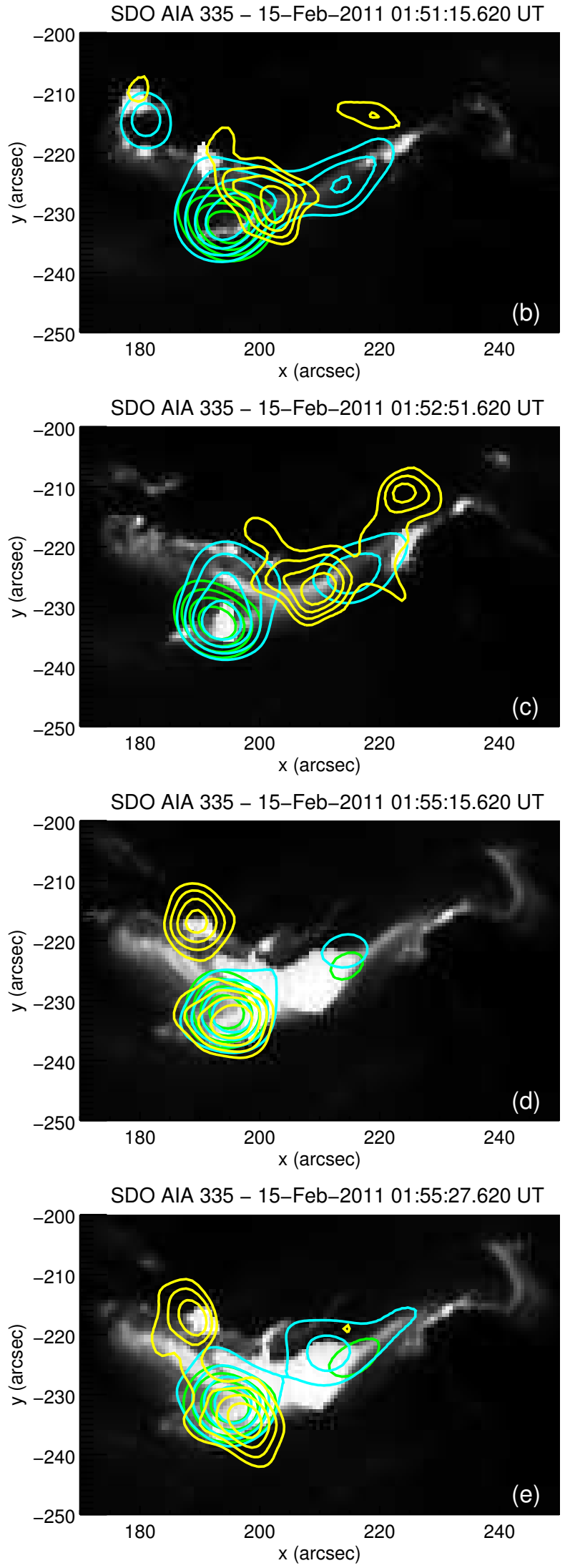

Fig. 6. SDO/AIA images at $335 \AA$ (grey scale), of a part of the active region 11158, on February 15, 2011. RHESSI X-ray contours 12-25 keV (green), 25-50 keV (cyan), and 50-100 keV (yellow) at 01:51:08, 01:52:10, 01:55:10, and 01:55:30 UT are overlaid (contour levels are the same as in Fig. 3).
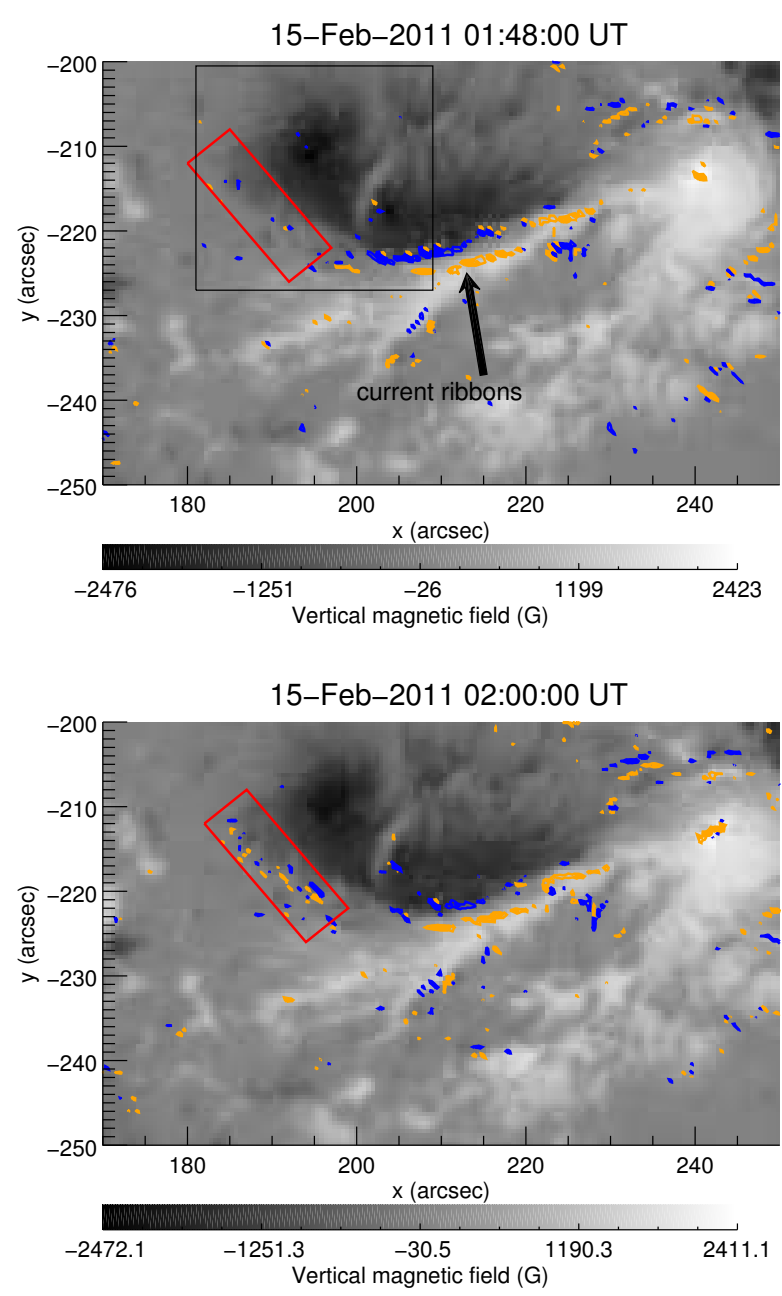

Fig. 7. Magnetic field maps (in grey scale) from SDO/HMI of a part of the active region 11158, on February 15, 2011, at 01:48:00 UT (top) and 02:00:00 UT (bottom), represented in the plane-of-sky. The orange and blue contours represent the positive and negative vertical electric current densities, respectively, with the magnitude greater than $100 \mathrm{~mA} / \mathrm{m}^{2}$. The black arrow in the top panel indicates the current ribbons extending from $195 \operatorname{arcsec}$ to 230 arcsec in the $x$-direction, and lying between -220 arcsec and -230 arcsec in the $y$-direction. The red box shows the primary difference between the two maps: in this box, the total negative vertical current density varies from $-974.9 \times 10^{9}$ A to $-1062 \times 10^{9} \mathrm{~A}$, and the total positive vertical current density varies from $+616.9 \times 10^{9} \mathrm{~A}$ to $+762.4 \times 10^{9} \mathrm{~A}$. Therefore, the total increase of vertical current density is of $232 \times 10^{9} \mathrm{~A}$ for the area of the red box. This represents an increase of $15 \%$ in the total current density in this area.

time of both X-ray and EUV sources at $335 \AA$. Only the $335 \AA$ can be used for this comparison since the emission is saturated at $94 \AA$ after 01:52 UT. In the last two images (intervals d and e) in which the configuration of HXR sources at $50-100 \mathrm{keV}$ is dramatically changed compared to interval (c), it should be noted that new arcade-like structures appeared in EUV between the two 50-100 keV sources.

\subsection{Comparison with the vertical electric current densities}

Figure 7 shows the combination of the vertical magnetic field and of the vertical currents for the field of view indicated by the black box in Fig. 1, i.e. the part where the X-ray emissions of 
S. Musset et al.: Hard X-ray emitting energetic electrons and photospheric electric currents
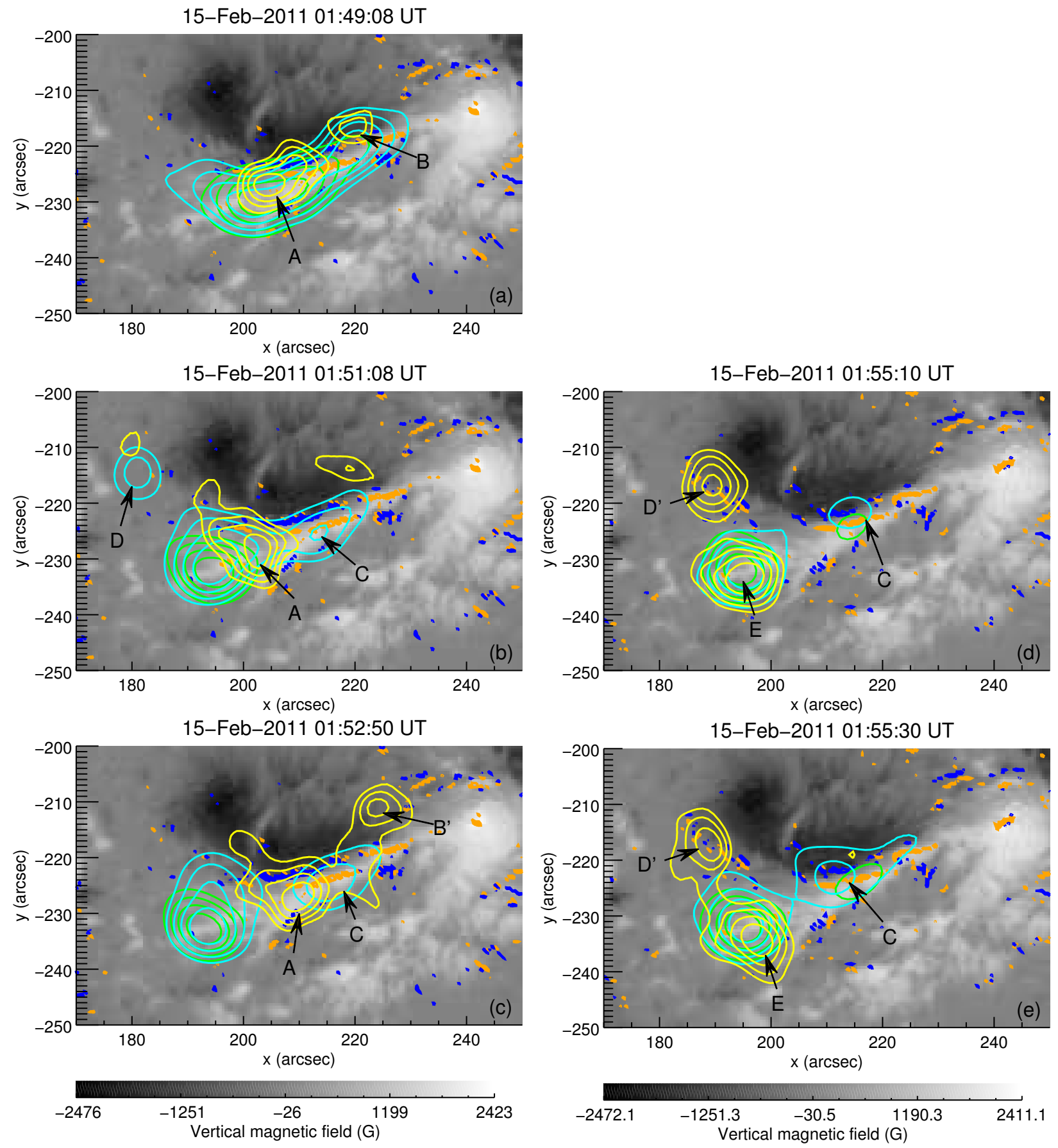

Fig. 8. Magnetic field maps (in grey scale) from SDO/HMI of a part of the active region 11158, on February 15, 2011, at 01:48:00 UT (left) and 02:00:00 UT (right). The orange and blue contours represent the positive and negative vertical electric current densities, respectively, with amplitude $>100 \mathrm{~mA} / \mathrm{m}^{2}$. The green, cyan, and yellow contours are the X-ray emissions (from RHESSI) at $12-25 \mathrm{keV}, 25-50 \mathrm{keV}$, and 50-100 keV, respectively, integrated between a) 01:49:00 and 01:49:16; b) 01:51:00 and 01:51:16; c) 01:52:42 and 01:52:58; d) 01:55:02 and 01:55:18; and e) 01:55:22 and 01:55:38 UT. X-rays have been imaged with the algorithm CLEAN, using collimators 2, 3, 4, 5, 6, 7, 8, and 9. The contours correspond to $60,70,80$, and $90 \%$ of the maximum for the X-ray emissions.

the flare come from. The improved spatial resolution of magnetograms allows very fine structures to be distinguished such as the current ribbons (Janvier et al. 2014), which are visible in Fig. 1 as the alignment of close positive and negative currents and which are shown by the black arrow in the top panel of Fig. 7. These current ribbons are present in both maps at 01:48 and 02:00 UT. We note that these narrow current ribbons have a fragmented appearance with many knots of high current densities distributed along the ribbons. The other noticeable feature in Fig. 7 (bottom) is the appearance of strong currents in the region marked by the red box. The current density in this region is increased by $15 \%$ between the two maps. This evolution of the currents has already been noted by Janvier et al. (2014).

Figure 8 shows the evolution of X-ray sources with time, overlaid on the vertical magnetic field and current density maps at the photospheric level obtained at 01:48:00 UT (left) and 
02:00:00 UT (right). Two main results can be drawn from this figure:

- Part of the elongated X-ray sources observed between 12 and $50 \mathrm{keV}$ in intervals (a), (b), and (c) overlay the current ribbons.

- The new X-ray source D' appearing at 50-100 keV in intervals (d) and (e) is located in the region where new vertical photospheric electric currents appeared between 01:48 and 02:00 UT.

The distance of the centroid of each X-ray source seen in Fig. 8 to the nearest current concentration has been estimated. Most of the HXR centroids are found to be within a distance lower than 4 arcsec from the closest part of a current ribbon, with the exception of two sources: the centroid of source $E$ (see images (d) and (e)), which is 8 to 12 arcsec from the nearest current ribbon, and the centroid of source B on image (b) which is at 6 arcsec from the nearest current ribbon. Figure 9 shows the X-ray images reconstructed with the visibility forward fit technique superimposed on the magnetic field and current density maps for intervals (b) and (c). The distance of the maximum of the elongated source (in the narrow energy ranges used in Sect. 3.1, see e.g. Table 1) to the nearest current concentration has also been estimated. This distance is found to decrease with photon energies, being of the order of 8 to 10 arcsec for energies below $26 \mathrm{keV}$ and below 6 arcsec for energies above $26 \mathrm{keV}$.

\section{Discussion and interpretation}

\subsection{How to interpret the spatial configuration of $X$-ray and EUV sources}

During the flare, the X-ray sources below $50 \mathrm{keV}$ have an elongated shape (more than 30 arcsec in length), cospatial with the EUV emission at $94 \AA$. A large proportion of the X-ray emission in the $25-50 \mathrm{keV}$ range is, however, mostly non-thermal (more than $75 \%$ of the total emission in this energy range). It can also be noted that the length of the source systematically increases with energy from $12-18 \mathrm{keV}$ to $40-60 \mathrm{keV}$ (see Table 1).

Such an elongated shape of the HXR emission as well as the increase in the source size with energy is not the standard situation. However, a few events have been reported in which non-thermal HXR emissions at $25-50 \mathrm{keV}$ had an elongated shape resulting from thick-target interactions in a dense coronal loop (see e.g. Veronig \& Brown 2004). The evolution with energy of HXR source sizes was also estimated by Xu et al. (2008) for several limb events on extended sources. They found, as in the present case, a slow increase in the source size with energy (the logarithmic slope found for several events is in the range $0.11 \pm 0.04-0.76 \pm 0.03$ ). They also predicted the evolution of the source size with energy when X-ray emission results from a thermal population of electrons produced at the apex of the loop or when X-ray emission results from non-thermal electrons accelerated in an extended region at the loop apex. They found that in the case of thermal emissions, the source size should decrease with energy, while it should increase in the case of nonthermal emissions. Combining these predictions with the present observations of X-ray source sizes (systematic increase with energy from $12-18 \mathrm{keV}$ to $40-60 \mathrm{keV}$ ), we therefore conclude that in this flare the $\mathrm{X}$-ray emissions in the $25-50 \mathrm{keV}$ range coming from the elongated sources arise primarily from non-thermal emissions (in agreement with the spectral analysis of Sect. 3.1).
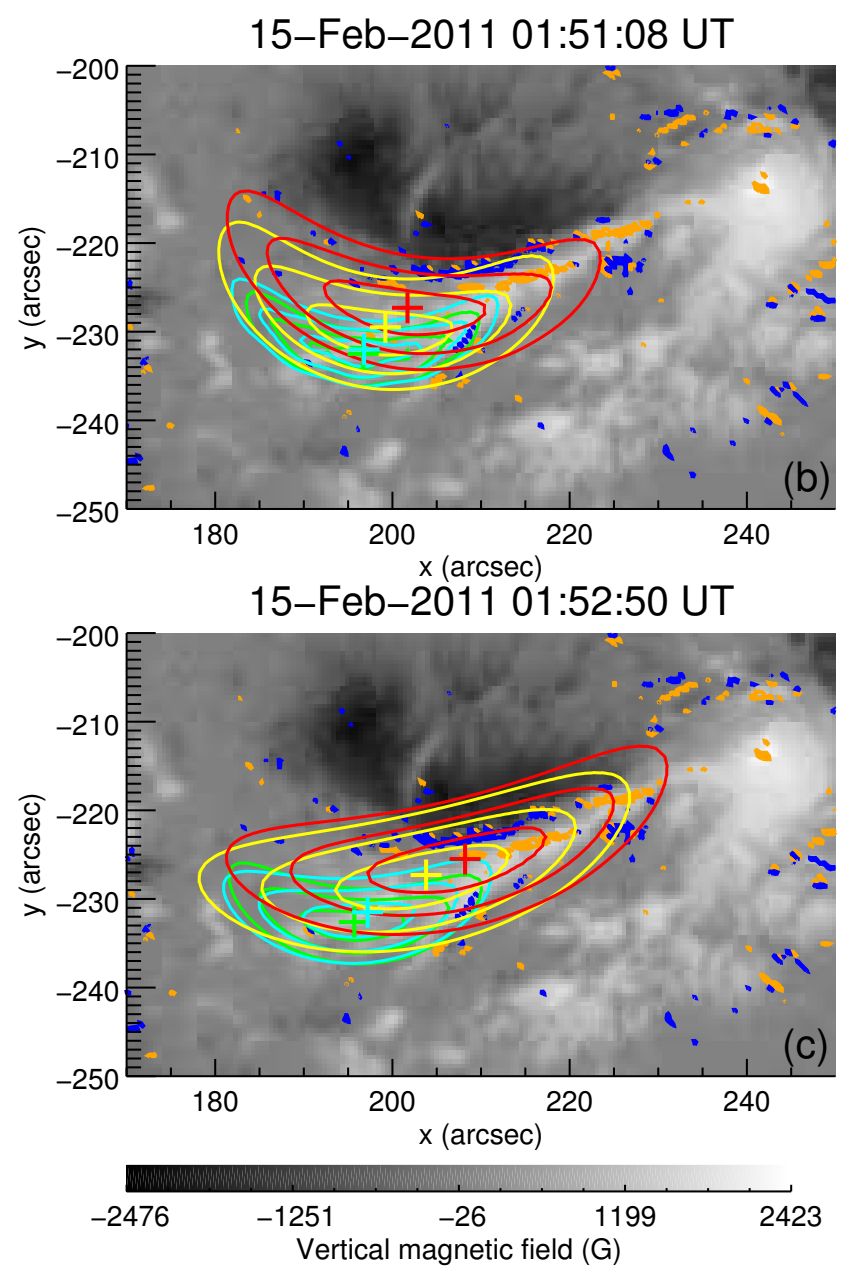

Fig. 9. Magnetic field map (in grey scale) from SDO/HMI of a part of the active region 11158, on February 15, 2011, at 01:48:00 UT. The orange and blue contours represent the positive and negative vertical electric current densities, respectively, with amplitude $>100 \mathrm{~mA} / \mathrm{m}^{2}$. The green, cyan, yellow, and red contours are the X-ray emission (from RHESSI) at 12-18 keV, $18-26 \mathrm{keV}, 26-40 \mathrm{keV}$, and 40-60 keV, respectively, integrated between b) 01:50:48 and 01:51:28 and c) $01: 52: 30$ and 01:53:10 UT. X-rays have been imaged with the Visibility Forward Fit technique using collimators 2, 3, 4, 5, 6, 7, 8, and 9 . The contours correspond to 50,70 , and $90 \%$ of the maximum for the X-ray emissions. The crosses are at the location of the maxima of the sources.

Furthermore following Xu et al. (2008) for the interpretation of the evolution of the source size with photon energy, it can be deduced that the acceleration region is extended: the size is found to be around 28 arcsec and the density of the loop of the order of $3 \times 10^{10} \mathrm{~cm}^{-3}$. These values are in good agreement with what has been found for a few events by Kontar et al. (2011) and Guo et al. (2012). In their work, Xu et al. (2008) modelled the transport of electrons on the basis of a standard collisional transport model. It was later shown by Kontar et al. (2014) that an increase of X-ray source size with energy can also be predicted in the context of a diffusive transport model of electrons, in which the turbulent pitch angle scattering of energetic electrons on magnetic fluctuations leads to an enhancement of the coronal HXR source relative to the footpoints. Comparing the predictions of this last model with our observations, the size of the acceleration region is found to be $22 \operatorname{arcsec}\left(1.6 \times 10^{4} \mathrm{~km}\right)$ and the electron mean free path of the order of $10^{9} \mathrm{~cm}$, which is 
in agreement with the results of Kontar et al. (2014) for another flare.

At higher energies $(>50 \mathrm{keV})$, the $\mathrm{X}$-ray emission is produced by a non-thermal population of electrons and the emissions come from more compact sources: sources $\mathrm{A}, \mathrm{B}$, and $\mathrm{B}^{\prime}$ in images (a) to (c) and sources $\mathrm{D}^{\prime}$ and $\mathrm{E}$ in images (d) and (e). While sources B and $\mathrm{B}^{\prime}$ appear to trace the impact of energetic electrons at the footpoint structures, the $50-100 \mathrm{keV}$ source labelled A in images (a), (b), and (c) is located in the middle of the elongated structure observed at lower energies. Its location is also very close to the position of the maximum of the elongated X-ray source in the 40-60 keV range. This highenergy source could be similar to coronal sources more easily observed in the case of limb events (Krucker et al. 2008, 2010; Chen \& Petrosian 2012; Su et al. 2013), but projected on the disk in our case. The combined location of coronal and looptop sources could trace the location where the main reconnection process occurs, i.e. above the loop in which electrons then propagate (see e.g. Su et al. 2013). In this context, the evolution of the location of sources A, B, and $\mathrm{B}^{\prime}$ between time intervals (a) and (c) most probably follow the evolution of the acceleration site. It must be noted that movement of X-ray footpoints are usually observed in the course of flares and are related to the magnetic reconnection process (see e.g. Fletcher et al. 2011, for a review). A more significant change in the configuration of HXR sources above $50 \mathrm{keV}$ is observed later in the flare (images (d) and (e)). Simultaneously with the hardening of the nonthermal electron spectra, new high-energy HXR sources appear (sources $\mathrm{D}^{\prime}$ and E) most probably located at the footpoints of an arcade of loops that appeared in EUV at the same time. These observations show that after $\approx 01 \mathrm{~h} 55$ UT, magnetic reconnection and the resulting plasma heating and particle acceleration occurs in a new structure. Finally, it can be seen that the position and orientation of the new EUV bright structure are consistent with those of the post-flare loops appearing in the 3D MHD simulation of the flare performed by Inoue et al. (2014).

\subsection{How to interpret the relation between $X$-ray emissions and photospheric vertical electric currents}

As discussed in Sect. 3.3, part of the elongated thermal and nonthermal X-ray sources (observed at energies below $50 \mathrm{keV}$ ) are located above the narrow and elongated current ribbons measured at the photospheric layer. These elongated X-ray structures were identified in the previous section as being produced in the corona by electrons injected from an extended acceleration region located close to the apex of the magnetic structures. At energies above $50 \mathrm{keV}$, the $\mathrm{X}$-ray source $\mathrm{A}$ is located very close (in projection) to the main current ribbon (shown by a black arrow in the top panel of Fig. 7) revealing the close association between the location of the acceleration region and the location of the electric current sheets.

As has been discussed in Janvier et al. (2014), the photospheric current ribbons (closely related to the flare EUV ribbons) are the tracers at the photospheric boundary of the electric current sheets present in the coronal volume. These current layers are themselves formed in regions of strong gradients of magnetic connectivity (see Fig. 7 in Janvier et al. 2014; and Zhao et al. 2014 for the computation of QSLs in the region) known to be the preferred locations where reconnection can occur (see e.g. Demoulin et al. 1996). As a consequence, plasma heating and particle acceleration can be produced in these regions. This explains why part of the thermal and non-thermal X-ray emissions produced in the coronal elongated sources are found above the photospheric current ribbons that trace the footprints of the coronal current layers.

At energies above $50 \mathrm{keV}$, the apparition of the X-ray source $\mathrm{D}^{\prime}$ is accompanied with the appearance of cospatial photospheric currents in the same time interval. We note that the observation of the current density evolution has to be carefully examined since polarization measurements during flares can be biased, and some of the observed features could result from polarization artefacts due to the impact of non-thermal particle beams (see Hénoux \& Karlický 2013, and references therein) or induced by resonant scattering due to radiation anisotropies at the edges of flare ribbons (Štěpán \& Heinzel 2013). Janvier et al. (2014) discussed this issue for this specific event. They cautiously analysed the vector magnetic field in the active region and concluded that the changes in the electric current density are not artefacts due to polarization effects. They argue that the horizontal fields (from which vertical electric currents are derived) are consistent in both maps at 01:36 UT and 02:00 UT with a signal free of artefacts given the smooth rotation of the magnetic field in this region. Figure 10 (based on Fig. 4 in Janvier et al. 2014) clearly shows a coherent direction of the horizontal magnetic field that varies smoothly within the field of view. Moreover, the curvature of the horizontal field lines increases between 01:48 and 02:00 UT, which is responsible for the increase of the observed vertical current (see also the observations by Petrie 2013). Finally, the increase in the current density shown in Fig. 7 is persistent, as can be seen in Fig. 10. This is a very strong argument against artefacts due to energetic particle precipitation or flare radiation anisotropies, because at 02:48 UT the GOES $\mathrm{X}$-ray flare has largely decayed. We thus reach the same conclusion as Janvier et al. (2014): the increase in the photospheric currents in the black box of Fig. 10 is not due to a polarization artefact but reflects real changes in the horizontal magnetic field and is thus real.

In conclusion, the appearance of the new HXR source $\mathrm{D}^{\prime}$ and the increase in the same region and in the same time interval of the photospheric currents are linked. The increase in the photospheric currents can be interpreted as a response at the photospheric layer of the change in magnetic topology and current systems in the corona induced by reconnection (see Janvier et al. 2014). As particle acceleration is also a consequence of the magnetic reconnection in the corona, it naturally explains the close temporal and spatial association between locally enhanced X-ray emission and increase in the same region and at the same time of photospheric currents: both result from the same phenomenon, namely magnetic reconnection in the localized coronal current sheets. Finally, it should be noted that such a related evolution of X-ray sources and electric currents in the course of a flare has not been reported previously, given the low cadence of magnetic field measurements before SDO/HMI. However, even in the present case, it is clear that the cadence to derive magnetic field maps (and current density maps) from HMI (12 min) is still too low to be able to track the evolution of electric currents on the timescales relevant to the evolution of X-ray sources (a few tens of seconds).

\subsection{Comparison with previous results}

As recalled in the introduction, the link between vertical electric currents and electron precipitation sites as diagnosed by 
01:48 UT

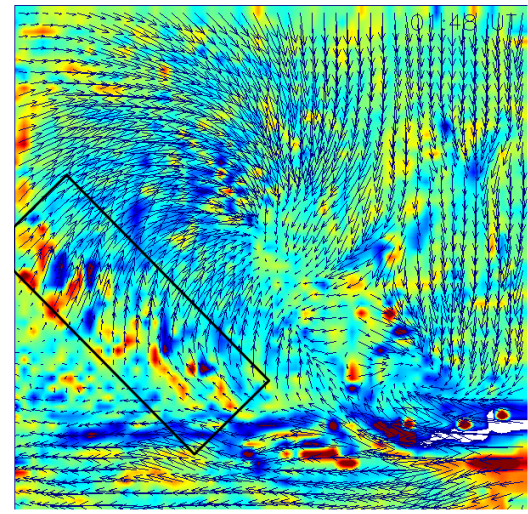

02:00 UT

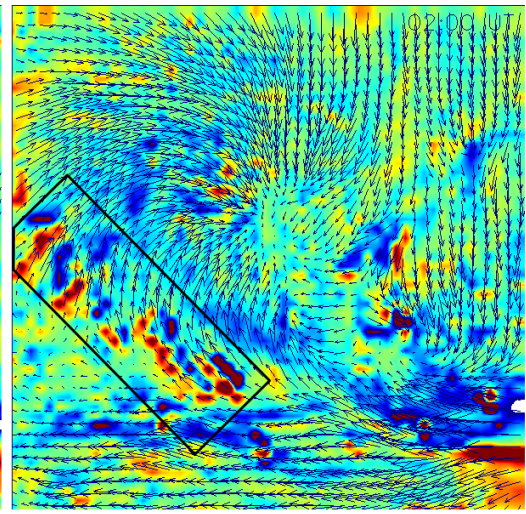

02:48 UT

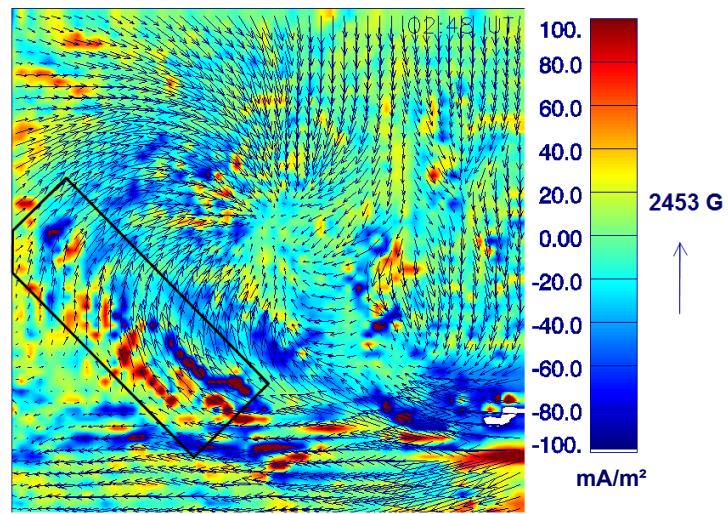

Fig. 10. Vertical current density (colour) and horizontal magnetic field (arrows, see scale), for the region indicated by the black box in Fig. 7 $\left(28^{\prime \prime} \times 26^{\prime \prime}\right.$ centred at $x=195$ arcsec and $y=-214$ arcsec), at 01:48 UT (left), 02:00 UT (middle), and 02:48 UT (right). The region where the current density increases by $15 \%$ between $01: 48$ and 02:00 UT is indicdated by the black rectangles.

HXR emissions at footpoints was investigated in the 1990s combining YOHKOH/HXT observations and vector magnetograph data from the Mees Solar Observatory (Li et al. 1997; Canfield et al. 1992). It was found that the electron precipitation sites as detected via the HXR footpoints are not exactly cospatial with the regions of highest vertical current densities but adjacent to the current channels. In this new study, using vector magnetograph data (and thus vertical electric maps) obtained from space (with an improved seeing) with a higher spatial resolution in combination with HXR images obtained at the same time and with improved image dynamics and spatial resolution, the link between electron precipitation sites and current concentrations has been revisited. These new observations confirm some of the previous results that HXR footpoints indicating electron precipitations sites are not exactly cospatial with the region of highest electric current densities. This is particularly the case in this flare of the footpoint sources B and E, which are at a distance of 6 to 12 arcsec from the nearest current ribbons in the present flare.

HXR footpoints can also be found at the same location as the current ribbons themselves. This is the case of the HXR footpoint $\mathrm{D}^{\prime}$ seen at energies above $50 \mathrm{keV}$ after 01:55 UT which is cospatial with new current ribbons which appeared themselves between 01:48 and 02:00 UT. It should be emphasized that the observation of such a combined evolution of HXR footpoints and electric currents is reported here for the first time. A complete interpretation of this observation is not within the scope of this paper aimed at observations, but would require a more complete study combining models of 3D reconnection in flares and particle acceleration.

In addition to HXR footpoint sources, a significant amount of the HXR emissions observed in this flare arise from an extended coronal source observed up to $60 \mathrm{keV}$. Such coronal HXR sources were not observed in the events studied by ( $\mathrm{Li}$ et al. 1997; Canfield et al. 1992). The comparison of coronal HXR sources with electric currents ribbons is then performed for the first time in this study. It is found that part of the thermal and non-thermal coronal sources just overlay the current ribbons. This should be further investigated in other events and interpreted in the context of combined models of 3D reconnection in flares and particle acceleration, but this clearly provides support to the models of particle acceleration in reconnecting current sheets (see e.g. Zharkova et al. 2011, for a review of acceleration models).

\section{Summary and conclusion}

This study is the first detailed comparison between the spatial distribution of photospheric vertical currents and of X-ray emission sites produced by hot plasma and accelerated electrons. For the first time, X-ray images and maps of electric currents (derived from vector magnetograms) were obtained at the same time with an improved time cadence for the observations of vector magnetograms (and derivation of electric current maps) which furthermore enables us to study the coupled evolution of the photospheric electric currents and the HXR emission sites in the course of the flare.

The main results obtained from this first study are the following:

- Parts of the thermal and non-thermal X-ray emissions produced in an extended coronal source overlay the elongated narrow current ribbons observed at the photospheric level.

- A new HRX source at energies above $50 \mathrm{keV}$ appears in the course of the flare in association with an increase of $15 \%$ of the photospheric current at around the same time and the same location. This shows a clear link between particle acceleration and reconnecting current sheets.

These two results can be qualitatively explained in the context of the commonly admitted scenario in which magnetic reconnection occurs at current-carrying QSLs in the corona with part of the energy released transferred to plasma heating and particle acceleration. Since X-ray (and EUV) emissions are signatures of plasma heating and of particle acceleration, and since photospheric currents can trace the footprints of the coronal currents embedded in QLSs, some spatial and temporal correlations can naturally be expected between X-ray (and EUV) emitting sites and photospheric current ribbons. In addition, the evolution of magnetic reconnection sites in the course of the flare may lead to two linked consequences: on the one hand, plasma heating and particle acceleration are produced at different locations, potentially leading to the appearance of not only new EUV postflare loops, but also of new X-ray sources; on the other hand, the change of magnetic topology due to the evolving reconnection process leads to an increase in the photospheric current densities at the same place and time (see also Janvier et al. 2014). These new results have been obtained so far on only one flare. A similar study should be further extended in the future to other X-class 
flares, to examine whether this relation between photospheric current ribbons and X-ray sources is systematically observed. This work furthermore shows that the evolution of horizontal magnetic fields and of derived photospheric vertical electric currents can be observed on timescales of 12 min during a strong flare. The cadence is, however, still too low to derive the detailed timing between the evolution of the fields and currents and the evolution of the energetic electron acceleration and interaction sites. Finally, this kind of study deserves future interpretation and modelling: in particular, tentatively coupling acceleration processes to 3D MHD models would allow the derivation of the location of the reconnecting current sheets and of their observed photospheric traces together with the location of HXR sources.

Acknowledgements. We thank Mikola Gordovskyy, Guillaume Aulanier, Miho Janvier, Anna Massone and Brigitte Schmieder for their useful comments, as well as the RHESSI team for providing free access to data. SDO data were made available courtesy of the NASA/SDO AIA and HMI science teams. The authors thank the anonymous referee for his suggestions which led to major improvements in this manuscript. Sophie Musset acknowledges the CNES and the LABEX ESEP $\left(\mathrm{N}^{\circ}\right.$ 2011-LABX-030) for the PhD funding, and thanks the French State and the ANR for their support through the "Investissements d'avenir" programm in the PSL* initiative (convention No. ANR-10-IDEX-0001-02).

\section{Appendix A: Spatial superposition of magnetic field, electric current density, and emissions from flares}

\section{A.1. Change of frame}

To compare the X-ray emissions with magnetic field and current density maps, the frame of reference must be carefully chosen. Indeed, the X-ray images are created in the Cartesian coordinates (plane-of-sky), whereas the magnetic maps were taken and derived in the heliographic system (heliographic plane, see Fig. A.1). We chose to use in the paper the Cartesian coordinates, since X-ray sources vary on timescales of the order of seconds, whereas the magnetic field maps are obtained at a cadence of $12 \mathrm{~min}$.

The relation between $(x, y)$, the Cartesian coordinates of a point and its heliographic coordinates $(L, b)$ is given by

$\left\{\begin{array}{l}x=\sin \left(L-L_{\mathrm{C}}\right) \cos (b) \\ y=\sin (b) \cos \left(B_{\mathrm{C}}\right)-\cos \left(L-L_{\mathrm{C}}\right) \cos (b) \sin \left(B_{\mathrm{C}}\right)\end{array}\right.$

with $L$ the longitude of the point relative to the central meridian, $b$ the latitude of the point, and $B_{\mathrm{C}}$ and $L_{\mathrm{C}}$ the latitude and longitude of the centre of the solar disk at the time of observation.

The change of frame also modifies the pixel size. In the case of an active region located near the disk centre, several approximations can be made and the pixel size is described by

$$
\left\{\begin{aligned}
p x_{\mathrm{car}} \approx & p x_{\text {helio }} \cos \left(L-L_{\mathrm{C}}\right) \\
p y_{\mathrm{car}} \approx & p x_{\mathrm{helio}}\left(\cos (b) \cos \left(B_{\mathrm{C}}\right)\right. \\
& \left.+\cos \left(L-L_{\mathrm{C}}\right) \sin (b) \sin \left(B_{\mathrm{C}}\right)\right)
\end{aligned}\right.
$$

with $\left(p x_{\text {car }}, p y_{\text {car }}\right)$ the pixel size in the Cartesian coordinates (in the plane-of-sky), and ( $\left.p x_{\text {helio }}, p y_{\text {helio }}\right)$ the pixel size in the heliographic coordinates (in the heliographic plane).

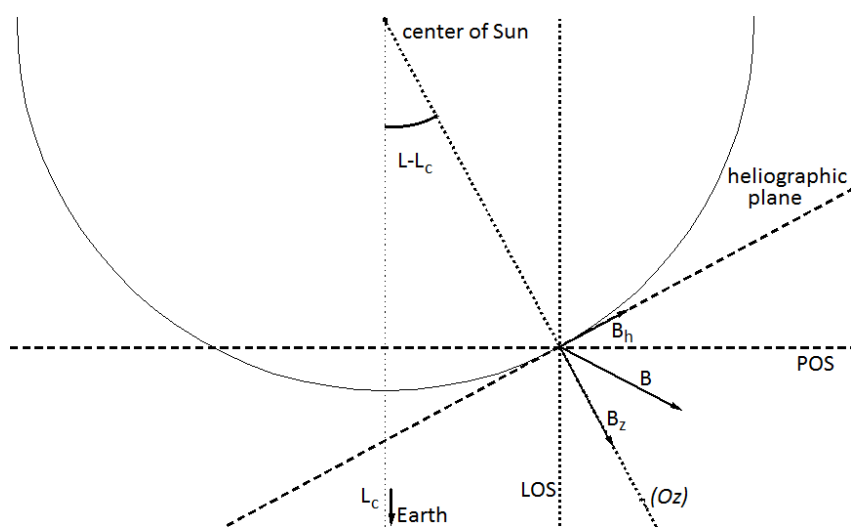

Fig. A.1. Heliographic coordinates: line emission used for spectropolarimetry comes from one layer of the Sun (circle). The magnetic field and electric current density are then calculated in the heliographic coordinates, as the drawing shows: the horizontal component is in the heliographic plane, which is tangent to the considered layer of the Sun, and the vertical component is perpendicular to that along the $(O z)$ axis. The $\mathrm{X}$-ray emissions are taken in the plane-of-sky (POS), i.e. in the plane perpendicular to the line of sight (LOS).

\section{A.2. Time gap between different observations}

The second problem when comparing X-ray emissions and magnetic field maps is the time gap between the different observations. We use the time of the X-ray images as a reference since $\mathrm{X}$-ray sources vary faster than the magnetic field, and also because we generally have more images in X-rays than maps of the magnetic field.

The time gap between the X-ray images and the magnetic maps must be taken into account because the rotation of the solar surface is not negligible. It has been corrected by setting the observation time of the X-ray image when looking for $B_{C}$ and $L_{C}$ in the ephemerids to use Eq. (A.1). We note that we used the get_sun procedure in the SolarSoft to get the ephemerids $\left(B_{\mathrm{C}}\right.$ and $L_{\mathrm{C}}$ at time of observation).

\section{References}

Aschwanden, M. J., Kosugi, T., Hanaoka, Y., Nishio, M., \& Melrose, D. B. 1999, ApJ, 526, 1026

Aschwanden, M. J., Sun, X., \& Liu, Y. 2014, ApJ, 785, 34

Aulanier, G., Pariat, E., \& Démoulin, P. 2005, A\&A, 444, 961

Bagalá, L. G., Mandrini, C. H., Rovira, M. G., Démoulin, P., \& Hénoux, J. C. 1995, Sol. Phys., 161, 103

Bommier, V., Landi Degl'Innocenti, E., Landolfi, M., \& Molodij, G. 2007, A\&A, 464,323

Canfield, R. C., Hudson, H. S., Leka, K. D., et al. 1992, PASJ, 44, L111

Canfield, R. C., de La Beaujardiere, J.-F., Fan, Y., et al. 1993, ApJ, 411, 362

Chen, Q., \& Petrosian, V. 2012, ApJ, 748, 33

de La Beaujardiere, J.-F., Canfield, R. C., \& Leka, K. D. 1993, ApJ, 411, 378

Demoulin, P., Henoux, J. C., Priest, E. R., \& Mandrini, C. H. 1996, A\&A, 308, 643

Demoulin, P., Bagala, L. G., Mandrini, C. H., Henoux, J. C., \& Rovira, M. G. 1997, A\&A, 325, 305

Fletcher, L., Dennis, B. R., Hudson, H. S., et al. 2011, Space Sci. Rev., 159, 19 Guo, J., Emslie, A. G., Kontar, E. P., et al. 2012, A\&A, 543, A53

Hagyard, M. J., Teuber, D., West, E. A., \& Smith, J. B. 1984, Sol. Phys., 91, 115 Hénoux, J. C., \& Karlický, M. 2013, A\&A, 556, A95

Hurford, G. J., Schmahl, E. J., Schwartz, R. A., et al. 2002, Sol. Phys., 210, 61 Inoue, S., Hayashi, K., Magara, T., Choe, G. S., \& Park, Y. D. 2014, ApJ, 788, 182

Janvier, M., Aulanier, G., Bommier, V., et al. 2014, ApJ, 788, 60

Kontar, E. P., Hannah, I. G., \& Bian, N. H. 2011, ApJ, 730, L22

Kontar, E. P., Bian, N. H., Emslie, A. G., \& Vilmer, N. 2014, ApJ, 780, 176

Krucker, S., Battaglia, M., Cargill, P. J., et al. 2008, A\&ARv, 16, 155

Krucker, S., Hudson, H. S., Glesener, L., et al. 2010, ApJ, 714, 1108 


\section{A\&A 580, A106 (2015)}

Leka, K. D., Canfield, R. C., McClymont, A. N., et al. 1993, ApJ, 411, 370 Leka, K. D., Barnes, G., Crouch, A. D., et al. 2009, Sol. Phys., 260, 83 Lemen, J. R., Title, A. M., Akin, D. J., et al. 2012, Sol. Phys., 275, 17

Li, J., Metcalf, T. R., Canfield, R. C., Wuelser, J.-P., \& Kosugi, T. 1997, ApJ, 482,490

Lin, Y., \& Gaizauskas, V. 1987, Sol. Phys., 109, 81

Lin, R. P., Dennis, B. R., Hurford, G. J., et al. 2002, Sol. Phys., 210, 3

Machado, M. E., Somov, B. V., Rovira, M. G., \& de Jager, C. 1983, Sol. Phys., 85,157

Mandrini, C. H., Demoulin, P., Rovira, M. G., de La Beaujardiere, J.-F., \& Henoux, J. C. 1995, A\&A, 303, 927

Moreton, G. E., \& Severny, A. B. 1968, Sol. Phys., 3, 282

Petrie, G. J. D. 2012, ApJ, 759, 50

Petrie, G. J. D. 2013, Sol. Phys., 287, 415

Romanov, V. A., \& Tsap, T. T. 1990, Sov. Astron., 34, 656
Scherrer, P. H., Schou, J., Bush, R. I, et al. 2012, Sol. Phys, 275, 207 Schmahl, E. J., Pernak, R. L., Hurford, G. J., Lee, J., \& Bong, S. 2007, Sol. Phys. 240, 241

Schou, J., Scherrer, P. H., Bush, R. I., et al. 2012, Sol. Phys., 275, 229

Schrijver, C. J., Aulanier, G., Title, A. M., Pariat, E., \& Delannée, C. 2011, ApJ, 738, 167

Smith, D. M., Lin, R. P., Turin, P., et al. 2002, Sol. Phys., 210, 33

Su, Y., Veronig, A. M., Holman, G. D., et al. 2013, Nat. Phys., 9, 489

Sun, X., Hoeksema, J. T., Liu, Y., et al. 2012, ApJ, 748, 77

Štěpán, J., \& Heinzel, P. 2013, ApJ, 778, L6

Vemareddy, P., Ambastha, A., \& Maurya, R. A. 2012, ApJ, 761, 60

Veronig, A. M., \& Brown, J. C. 2004, ApJ, 603, L117

Xu, Y., Emslie, A. G., \& Hurford, G. J. 2008, ApJ, 673, 576

Zhao, J., Li, H., Pariat, E., et al. 2014, ApJ, 787, 88

Zharkova, V. V., Arzner, K., Benz, A. O., et al. 2011, Space Sci. Rev., 159, 357 